\title{
Clinical and Functional Outcomes using the Kyocera Modular Limb Salvage System for Lower Extremity Reconstruction
}

\section{Donnel Alexis Ting Rubio}

University of the Philippines College of Medicine https://orcid.org/0000-0002-0716-7925

Katsuhiro Hayashi ( $\nabla$ khayashi830@gmail.com )

Kanazawa University https://orcid.org/0000-0001-8665-2154

\section{Norio Yamamoto}

Department of Orthopedic Surgery Kanazawa University

\section{Toshiharu Shirai}

Department of Orthopedics Kanazawa University

\section{Akihiko Takeuchi}

department of orthopaedic surgery Kanazawa university

\section{Hideji Katou}

Department of Orthopedic Surgery Kanazawa University

\section{Yu Aoki}

Department of Orthopedic Surgery Kanazawa University

\section{Takashi Higuchi}

Department of Orthopedic Surgery Kanazawa University

Ahmed Hamed Kassem Abdelaal

Department of Orthopedic Surgery Sohag University

Hiroyuki Tsuchiya

Department of Orthopedics Kanazawa University

\section{Research article}

Keywords: Clinical Outcome, Functional Outcome, Kyocera Modular Limb Salvage System, Limb Salvage Surgery

Posted Date: July 21 st, 2020

DOI: https://doi.org/10.21203/rs.3.rs-37321/v1

License: (c) (1) This work is licensed under a Creative Commons Attribution 4.0 International License. Read Full License 


\section{Abstract}

Background: Segmental metallic endoprostheses are widely used and important in reconstructive procedures of the lower limb. Most of the currently available endoprostheses are made in Europe or the United States of America and are too large for Asian knees. The Japanese Musculoskeletal Oncology Group developed a tumor prosthesis better suited for Asian skeletal anatomy, the Kyocera Modular Limb Salvage System (KLS).

Methods: We performed a retrospective review of the records of patients who underwent reconstruction using KLS implants. Thirty-five patients fulfilled the inclusion criteria, 20 having undergone limb salvage surgery for tumor and 15, joint arthroplasty. Functional outcomes were evaluated using the revised Musculoskeletal rating scale for the tumor group and the Japanese Orthopedic Association Knee Score for the arthroplasty group. Complications and survivorship of the implants were also assessed.

Results: At the latest follow-up, in the tumor group, 13 were alive with no evidence of recurrence, 6 died of disease, and 1 was alive with evidence of disease. The average MSTS scores on latest follow-up were 63 for the proximal femur, 67 for the distal femur, and 92 for the proximal tibia. The mean prosthesis survival was 28 months. Complications include 4 cases of aseptic loosening, 2 deep infections, 1 superficial infection, 1 valgus deformity, 2 periprosthetic fractures, 2 incidences of excessive polyethylene wear, and 2 local tumor recurrences.

In the arthroplasty group, 1 patient had a persistent low grade infection on the latest follow-up. The postoperative JOA score was 61 (mean). Complications included 3 cases of aseptic loosening, 2 deep infections, 2 implant failures, 1 superficial infection, 1 valgus deformity, and 1 soft tissue defect.

Conclusion: Early results of the KLS implant system are encouraging, with comparable clinical outcomes to other systems in the literature. A longer follow-up study with increased numbers of patients is necessary to further validate the functional outcomes and prosthesis survival using the KLS system for limb salvage surgery.

\section{Background}

Segmental metallic endoprostheses are becoming increasingly important in limb reconstruction following tumor surgery and revision total joint replacement [1-2]. However, this type of reconstruction does not come without risks. Postoperative complications can occur and these include infection, aseptic loosening, joint instability, and structural and implant-related failures. [1, 3-4]

Infection, aseptic loosening and instability can be devastating complications and are considered the 3 most common causes of morbidity after primary and revision joint arthroplasty and in limb salvage surgery using an endoprosthesis [1, 5-10]. 
Most of the currently available endoprostheses are made in Europe or the United States of America and are designed for Caucasians patients. A problem noted with these implants is that they can be too large for Asian knees. In Japan, the Japanese Musculoskeletal Oncology Group developed a tumor prosthesis better matching Asian skeletal anatomy, the Kyocera Modular Limb Salvage System (KLS system). It is designed with a semi-rotating hinge joint with the metallic parts made of titanium alloy [11].

The purpose of this study is to evaluate the clinical and functional outcomes and complications of using the KLS system implants for limb salvage surgery and in primary and revision joint arthroplasty.

\section{Methods}

A retrospective review of the records of patients who underwent reconstruction using the KLS implants was done. Data were obtained from the computer database of the Department of Orthopedic Surgery, Kanazawa University, Japan. This study was approved by the Institutional Review Board of the Kanazawa University Hospital, Kanazawa, Japan, and this study complied with the ethical standards outlined in the Declaration of Helsinki

Patients who underwent reconstruction using the KLS system implant (whether for reconstruction after limb salvage surgery or for primary/revision arthroplasty) and with at least 1 year follow-up were included in the study. All patients underwent surgery by the Department of Orthopedic Surgery at Kanazawa University Hospital.

Over a period of 5 years (2008-2013), a total of 48 patients underwent reconstruction using titanium implants. We limited our study, however, to those who specifically underwent reconstruction using the KLS endoprosthesis. Thirty-five patients fulfilled the inclusion criteria, 20 from limb salvage surgery and 15 from arthroplasty, and were assigned to the Tumor or Arthroplasty group for analysis.

Complications for both groups were assessed. Functional outcomes were also evaluated using the revised Musculoskeletal Tumor Society (MSTS) Score for the tumor group and the Japanese Orthopedic association (JOA) score for the arthroplasty group [12-13]. Range of motion (ROM) was documented using a basic goniometer.

Survivorship of the KLS implant was also evaluated. Implant failure was defined as one requiring an unplanned revision of a failed portion of the prosthesis, a periprosthetic fracture needing fixation, softtissue reconstruction needed to restore joint stability, and endoprosthetic removal without revision and amputation [1].

Shirai et.al. [14] reported the efficacy and antimicrobial properties of the iodine coated titanium implants; all the KLS implants in this study were iodine coated to deter or treat periprosthetic infection.

\section{Results}


A total of 35 patients were included and evaluated in the study (Table 1).

\section{Tumor Group}

The most common indication for reconstruction using the KLS system in the tumor group was osteosarcoma and the most common bone involved was the distal femur (Table 1). Nine patients underwent wide excision and immediate reconstruction using the iodine coated KLS endoprosthesis and 11 underwent a two-staged revision, with the initial surgery being a wide excision and reconstruction using other techniques (recycled bone by liquid nitrogen freezing and reconstruction with either an intramedullary nail, plate, or non-iodine coated endoprosthesis) and then a second stage revision using the iodine coated KLS endoprosthesis from complications from the index surgery ( 6 infections, 2 local recurrences, 1 periprosthetic fracture, 1 nonunion and collapse of lateral condyle and 1 implant failure). Eight patients underwent cemented fixation while 11 patients received the cementless type of KLS implant. One patient underwent total femur replacement.

On the latest follow-up, 13 were alive with no evidence of recurrence, 6 died of disease, and 1 was alive with evidence of disease. The average MSTS scores on latest follow-up were 63 for the proximal femur (range: 40 to 100), 67 for the distal femur (range: 40 to 100) and 92 for the proximal tibia (range: 80 to 100). Mean ROM was 1 to $87^{\circ}$ flexion arc (range 0-10 degrees extension and 45-120 degrees flexion) for the knee and 90 degrees flexion, 60 degrees abduction, 0 adduction and 0 external rotation for the hip. The mean prosthesis survival was 28 months (range: $12-56$ months).

Modes of failure for the Tumor group are summarized in Table 2. Ten revisions of the iodine coated titanium implant were done to treat these complications ( 3 cases of aseptic loosening, 2 deep infections, 1 valgus deformity, 2 cases of excessive polyethylene wear, 1 local tumor recurrence). For the two patients who had local recurrence, one underwent total femur replacement for a local recurrence of a distal femur osteosarcoma and the other one underwent hind quarter amputation due to a diffuse local recurrence of the distal femur osteosarcoma. The limb salvage rate was 95\% (19/20).

\section{Arthroplasty Group}

The knee was the affected joint in all patients. Three patients underwent immediate reconstruction using the iodine coated KLS endoprosthesis after undergoing debridement and application of cement spacer as an index surgery (2 TB arthritis and 1 osteomyelitis) and 12 patients underwent 2-stage revision, the index case being total knee arthroplasty, which became infected and required revision using iodine coated KLS endoprosthesis after the initial infection was controlled. Five patients had cemented implants and 10 had the cementless type of the KLS prosthesis.

At latest follow-up, 14 patients were infection free and 1 had a consistent low grade infection (suppressed with oral antibiotics). Post-operative JOA score (mean) was 61 (range: 30 to 85) and mean ROM was 3 degrees to 81 degrees of knee flexion (range: 0 to30 degrees extension and 40 to 105 flexion). Mean prosthesis survival was 27 months (range: 12 to 62 months). 
Modes of failure for the Arthroplasty group are summarized in Table 3. Two of the three cases of aseptic loosening were at the side plate and screws of the cementless KLS implant. Five revision surgeries were necessary to treat these complications ( 2 cases of aseptic loosening, 2 implant failures and 1 infection)

\section{Discussion}

Segmental metallic prostheses have several advantages over biologic reconstruction in limb salvage surgeries, which include initial reliability with lower complication rates, ready availability and immediate stability allowing rapid return to full weight bearing with predictable function. However, long term risks for infection, local tumor recurrence, aseptic loosening, and implant failure are significant concerns for tumor surgeons [15].

Implant-related complications are still common in Orthopedic surgery and pose a serious problem. Several innovations were developed to counter or minimize these complications. One of these is the development of an endoprosthesis better matched to Asian knees by the Japanese Musculoskeletal oncology group. Nakamura et. al. [11] reported that the average MSTS score for the KLS system was 21.8 (72.5\%). The complication rate was $34 \%$ (28 out of 80 patients), infection rate $8.5 \%$ (7 out of 82 ) and aseptic loosening rate $6 \%$ (5 out of 82 ). In another study, Tome et. al. [16] investigated the clinical outcome of the KLS system for malignant bone tumors of the femur. The 3 year prosthesis survival rate was $87.5 \%$ and the 5 year survival rate was $58.3 \%$. The mean MSTS score was $81 \%$ and no prosthesisrelated complications were noted.

This implant was further improved by coating it with iodine to prevent and/or treat periprosthetic infection. Tsuchiya et.al. [17] performed a clinical trial on the efficacy and safety of the iodine coated implants. A total of 222 patients with postoperative infection or compromised states were treated using iodine coated titanium implants. The mean age was 49 and the mean follow-up was 18 months. lodine coated implants were used in 158 patients to prevent infection and 64 patients to treat active infections. In the prevention group, $3(1.9 \%)$ patients developed infection, however, all 3 recovered without implant removal. In the treatment group, infection was eliminated in all patients. Despite using iodine coated implants, no abnormalities related to the thyroid gland were detected and good bony ingrowth was noted.

In our series, the most common complications for both groups were infection and aseptic loosening, which are consistent with published reports [1, 5-10].

It is reported that the infection rate after primary total joint replacement ranges from $0.2 \%$ to $4 \%[5,7,18]$. For revision total joint arthroplasty, the risk of infection was 10 -fold higher than for patients undergoing primary total knee arthroplasty [7]. In one study, the incidence of infection after revision joint surgery was $46 \%[6]$.

In our series, the infection rate after revision total knee arthroplasty was lower, $17 \%$ (2 of 12 ) and only one patient required removal of the implant for re-revision. All of the single-staged reconstruction patients were infection free at latest follow-up. 
In limb salvage procedures, the infection rates are higher because patients are often immunocompromised. These patients undergo long and complex reconstructive procedures, often with large areas of dead space created after removal of bone and soft tissue, and have large wounds that often lack soft tissue coverage [19]. The incidence of infection after limb salvage surgery and endoprosthesis reconstruction ranges from $1 \%$ to $36 \%$ [20].

In a multicenter study reviewing the modes of failure after using an endoprosthesis for tumor reconstruction, Henderson [1] et al. reported that infection is the most common cause of failure after surgical reconstruction with an incidence of $34 \%$. In our series, the infection rate was $10 \%$ ( 2 of 20 cases). Both patients required removal of the implant and revision using an iodine-coated endoprosthesis.

The incidence of aseptic loosening after primary joint arthroplasty ranges from $19 \%$ to $31 \%$, and for revision arthroplasty, it was reported to be $19 \%[6,10]$. Contributing factors for loosening include patient specific factors (e.g. activity level, body mass index and body weight, gait mechanics, etc.), surgeon specific factors (e.g. surgical approach, component positioning, soft-tissue balancing, etc.) and implant specific factors (e.g. implant design, material, bearing couples, etc.) [21]. For limb salvage surgery and reconstruction using a tumor endoprosthesis, the incidence of aseptic loosening ranges from $5 \%$ to $27 \%$ [20]. Factors contributing to aseptic loosening of tumor endoprostheses include the extended length of the prosthesis and constrained joint design, both of which create high bending stresses at the prosthesisbone interface [1].

The incidence of aseptic loosening was $20 \%$ for both our study groups and was within the reported incidence $[6,10,20]$. All cases of aseptic loosening were noted in the cementless KLS prosthesis subgroup. Most of the causes of aseptic loosening in our series were attributed to the side plates/screw fixation of this implant; 1 of 4 in the tumor group and 2 of 3 in the arthroplasty group. The femur is not tubular over its distal aspect and the endoprosthesis side plate follows this tapered end of the distal femur. The cortical fixation by the screws, however, is not rigid enough to maintain the straight side plate compressed along the cortical taper of the distal femur and combined with a high stress area; micromotion occurs at this interface postoperatively. This usually happens before any bony in-growth occurs thus, loosening results (Figure 1).

For the tumor group, the mean MSTS scores were 63 for the proximal femur, 67 for the distal femur and 92 for the proximal tibia and it is comparable to average reported MSTS from the literature $(60 \%-90 \%)$ $[11,20]$. The best results were noted in patients who underwent proximal tibia reconstruction with the worst being in the proximal femur. These results are consisted with the study done by Gosheger et.al [22]. The 2 year prosthesis survival in our study was $79 \%$. In the literature, reported 5 year survival of the lower extremity prostheses ranges from $69 \%$ to $87 \%[16,20]$. Currently, it is difficult to compare our results to published reports because of our short follow-up.

In the Arthroplasty group, the post-operative mean JOA score was 61 and the 2 year prosthesis survival was $84 \%$. At present, there are no available reports on functional outcomes of the KLS system used for non-tumor cases. 
No knee instability was noted in either group on the latest follow-up. This may be attributed to the constrained design of the KLS implant. Constrained knee prostheses provide great inherent stability to the knee by means of a link between the femoral and tibial components which prevents varus-valgus and translational stresses [2].

Limitations of our study include the small number of patients studied and the limited follow-up. To more accurately determine the functional outcomes and prosthesis survival, an increased population size and longer follow-up are needed.

\section{Conclusion}

Early results using the KLS implant system are encouraging, with comparable functional and clinical outcomes to those reported in the literature. The cementless type of distal femur implant may have a higher incidence of aseptic loosening compared with the cemented type. A long term follow-up study and increased number of patients are necessary to further validate the functional outcomes and prosthesis survival using this system for limb salvage surgery.

\section{Declarations}

\section{Ethics approval and consent to participate}

This study was approved by the Institutional Review Board of the Kanazawa University Hospital, Kanazawa, Japan, and this study complied with the ethical standards outlined in the Declaration of Helsinki. Written informed consent was obtained from all participants.

\section{Consent for Publication}

Written informed consent was obtained from all participants.

\section{Availability of Data and materials}

The datasets used and analyzed during the current study are available from the corresponding author on reasonable request.

\section{Competing interests}

The authors declare that they have no competing interests.

\section{Funding}

Not applicable

\section{Author's Contribution}


DAR, YA, TH, AHA and HK collected and organized the data and helped in the analysis of the data. DAR, $\mathrm{KH}, \mathrm{NY}, \mathrm{TS}, \mathrm{AT}$, and $\mathrm{HT}$ analyzed the data and help edit the manuscript. DAR wrote the manuscript. All authors read and approved the final manuscript.

\section{Acknowledgement}

Not Applicable

\section{Abbreviations}

JOA score - Japanese Orthopedic Association score

KLS system - Kyocera Modular Limb Salvage System

MSTS score Musculoskeletal Tumor Society score

ROM - Range of Motion

TB arthritis- Tuberculous Arthritis

\section{References}

1. Henderson, E, Groundland J, Pala E, Dennis J, Wooten R, Cheong D, al. (2011) Failure Mode Classification for Tumor Endoprostheses: Retrospective Review of Five Institutions and a literature review. J Bone Joint Surg Am. 93: 418-29. DOI: 10.2106/JBJS.J.00834

2. Sanguineti F, Mangano T, Formica M, Franchin F. (2014) Total Knee Arthroplasty with rotating-hinge endo-model prosthesis: clinical results in complex primary and revision surgery. Arch Orthop Trauma Surg. 134(11): 1601-7. DOI: 1007/s00402-014-2061-1

3. Barrack R, Lyons T, Ingraham R, Johnson J. (2000) The use of modular rotating hinge component in Salvage Revision Total Knee Arthroplasty. J Arthroplasty. 15(7): 858-66. DOI:1054/arth.2000.9056

4. Pradhan NR, Bale L, Kay P, Porter ML. (2004) Salvage Revision total knee replacement using endomodel rotating hinge prosthesis. Knee. 11(6): 469-73. DOI:1016/j.knee.2004.03.001 
5. Pulido L, Ghanem E, Joshi A, Purtill J, Parvizi J. (2008) Periprosthetic Joint Infection: The Incidence, Timing, and Predisposing Factors. Clin Orthop Relat Res 466: 1710-1715 DOI:1007/s11999-0080209-4

6. Suarez J, Griffin W, Springer B, Fehring T, Mason JB, et.al. (2008) Why do revision knee arthroplasties fail? J Arthroplasty. 23(6 suppl 1): 99-103. DOI:1016/j.arth.2008.04.020

7. Mortazavi SMJ, Schwartzenberger J, Austin M, Purtill J, Parvii J. (2010) Revision Total Knee Arthroplasty Infection Incidence and Predictors. Clin Orthop Relat Res 468: 2052-2059. DOI: 1007/s11999-010-1308-6

8. Jeys LM, Grimer RJ, Carter SR, Tillman RM. (2005) Periprosthetic infection in Patients Treated for an Oncologic Condition. J Bone Joint Surg Am. 87: 842-849. DOI: 2106/JBJS.C.01222

9. Bickels, J, Wittig JC, Kollender Y, Henshaw R, Kellar-Graney K, et.al. (2002) Distal Femur Resection with Endoprosthetic Resection. A long term follow-up Study. Clin Orthop Relat Res. (400): 225-35. DOI: 1097/00003086-200207000-00028

10. Schroer WC, Berend KR, Lombardi AV, Barnes CL, Bolognesi MP, et.al. (2013) Why are total knees failing today? Etiology of total knee revision in 201 and 2011. J Arthroplasty. 28(8 suppl): 116-9. DOI: 1016/j.arth.2013.04.056

11. Nakamura T, Matsumine A, Uchida A, Kawai A, Nishida Y, et.al. (2014) Clinical outcomes of Kyocera Modular Limb salvage system after resection of bone sarcoma of the distal part of the femur: the Japanese Musculoskeletal Oncology Group Study. Int Orthop. 38(4): 825-30. DOI:1007/s00264-0132151-7

12. The Japanese Orthopaedic Association Japanese Knee Society. (1994) Assessment criteria for knee diseases and treatments. Tokyo: Kanehara. 
13. Enneking W, Dunham W, Gebhardt M, Malawar M, and Pritchard. (1993) A System for the Functional Evaluation of Reconstructive Procedures After Surgical Treatment of Tumors of the Musculoskeletal System. Clin Orthop Relat Res Vol 286. PMID: 8425352

14. Shirai T, Tsuchiya H, Nishida H, Yamamoto N, Watanabe K, et. al. (2014) Antimicrobial megaprostheses supported with iodine. J Biomater Appl. 29(4): 617-23. DOI: $1177 / 0885328214539365$

15. Jeys L, Grimer R. (2009) The long term risks of infection and amputation with limb salvage surgery using endoprostheses. In: Tunn E, editor. The treatment of Bone and Soft Tissue Sarcoma. SpringerVerlag Berlin Heidelberg. Pp. 75-84. DOI: 1007/978-3-540-77960-5_7

16. Tsuchiya H, Shirai T, Nishida H, Murakami H, Kabata T, et.al. (2012) Innovative antimicrobial coating of titanium implants with lodine. J Orthop Sci. 17: 595-604. DOI: 1007/s00776-012-0247-3

17. Tome Y, Maehara H, Tanaka K, Kanaya F. (2010) Clinical Outcome of Malignant Bone Tumor of the Femur using KLS System. Orthopedics and Traumatology Vol. 59 no. 3: 476-480. https://doi.org/10.5035/nishiseisai.59.476

18. Blom AW, Taylor AH, Pattison G, Whitehouse S, Bannister GC. (2003) Infection after total hip arthroplasty The Avon Experience. J Bone Joint Surg [Br] 85-B: 956-9. DOI: 10.1302/0301.620X.85B7.14095

19. Racano A, Pazionis T, Farrokhyar F, Deheshi B, Ghert M. (2013) High infection rate outcomes in Longbone Tumor Surgery with endoprosthetic reconstruction in adults: a systematic review. Clin Orthop Relat Res. 471 (6): 2017-27. DOI: 1007/s11999-013-2842-9

20. Balke M, Ahrens H, Streitbürger A, Gosheger G, Hardes J. (2009) Modular endoprosthetic reconstruction in malignant bone tumors: Indications and Limits. In: Tunn E, editor. The treatment of Bone and Soft Tissue Sarcoma. Springer-Verlag Berlin Heidelberg. pp. 39-50. DOI: 1007/978-3-54077960-5_4 
21. Tsao K, Jones L, Lewallen D. (2008) What patient and surgical factors contribute to implant wear and osteolysis in total joint arthroplasty? J Am Acad Orthop Surg. 16(Suppl1): S7-S13.

DOI:5435/00124635-200800001-00004

22. Gosheger G, Gebert C, Ahrens H, Streitbuerger A, Winkelmann W, Hardes J (2006) Endoprosthetic reconstruction in 250 patients with sarcoma. Clin Orthop Relat Res 450:164-171.

DOI: 10.1097/01.blo.0000223978.36831.39

\section{Tables}

Table 1 - Summary of Demographics for the Tumor and Arthroplasty Group

\begin{tabular}{|c|c|c|}
\hline & Tumor Group $(\mathrm{N}=20)$ & $\begin{array}{l}\text { Arthroplasty } \quad \text { Group } \\
(\mathrm{N}=15)\end{array}$ \\
\hline Gender & $\begin{array}{l}\text { Male }=12 \\
\text { Female }=8\end{array}$ & $\begin{array}{l}\text { Male }=7 \\
\text { Female }=8\end{array}$ \\
\hline Mean Age & 44 (16 to 80 years old) & 73 (49 to 85$)$ \\
\hline $\begin{array}{l}\text { Mean } \\
\text { Follow-up }\end{array}$ & 39 months (12 to 73 months) & $\begin{array}{l}28 \text { months }(13 \text { to } 62 \\
\text { months })\end{array}$ \\
\hline Diagnoses & $\begin{array}{l}\text { Osteosarcoma }=14 \\
\text { Malignant Fibrous Histiocytoma }=2 \\
\text { Metastatic Bone Disease }=2 \text { (Breast Cancer and Renal Cell } \\
\text { Carcinoma) } \\
\text { Giant Cell Tumor }=1 \\
\text { Desmoplastic Fibroma }=1\end{array}$ & $\begin{array}{l}\text { Degenerative } \\
\text { Osteoarthritis = 9 } \\
\text { Tuberculous Arthritis = } 2 \\
\text { Septic Arthritis = } 1 \\
\text { Rheumatoid Arthritis = } 1 \\
\text { Osteomyelitis = 1 } \\
\text { Previous trauma = 1 }\end{array}$ \\
\hline $\begin{array}{l}\text { Affected } \\
\text { region }\end{array}$ & $\begin{array}{l}\text { Proximal Femur }=6 \\
\text { Distal Femur }=10 \\
\text { Proximal Tibia }=4\end{array}$ & Knee $=15$ \\
\hline
\end{tabular}

Table 2 - Summary of Failure Modes for the Tumor Group 


\begin{tabular}{|c|c|}
\hline Mechanical Complications & ( N ) \\
\hline Aseptic Loosening & 4 \\
\hline Valgus Deformity & 2 \\
\hline Periprosthetic Fracture & 2 \\
\hline Excessive Polyethylene wear & 2 \\
\hline Nonmechanical Complications & \\
\hline Deep Infection & 2 \\
\hline Local Recurrence & 2 \\
\hline Superficial Infection & 1 \\
\hline
\end{tabular}

Table 3 - Summary of Failure Modes for the Arthroplasty Group

\begin{tabular}{|c|c|}
\hline Mechanical Complications & ( N ) \\
\hline Aseptic Loosening & 3 \\
\hline Implant Failure & 2 \\
\hline Valgus Deformity & 1 \\
\hline Nonmechanical Complications & \\
\hline Deep Infection & 2 \\
\hline Soft Tissue defect & 1 \\
\hline Superficial Infection & 1 \\
\hline
\end{tabular}

\section{Figures}




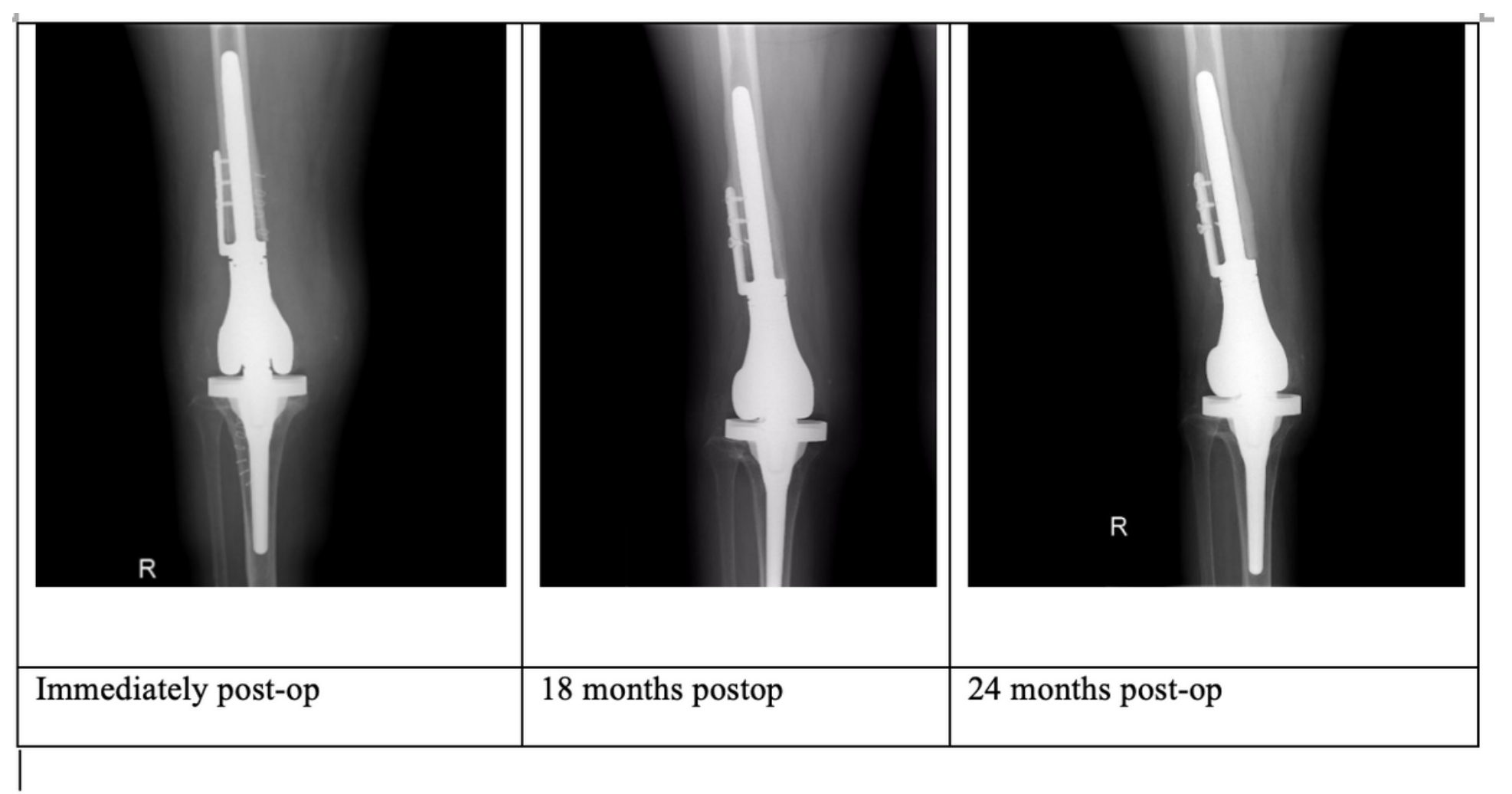

Figure 1

Aseptic Loosening of Uncemented KLS Tumor prosthesis 